\title{
SOME BILATERAL GENERATING FUNCTIONS INVOLVING THE ERKUŞ-SRIVASTAVA POLYNOMIALS AND SOME GENERAL CLASSES OF MULTIVARIABLE POLYNOMIALS
}

\author{
S. GABOURY, R. TREMBLAY AND M. A. ÖZARSLAN
}

\begin{abstract}
Recently, Liu et al. [Bilateral generating functions for the Erkuş-Srivastava polynomials and the generalized Lauricella function, Appl. Math. Comput. 218 (2012), pp. 7685-7693] investigated some various families of bilateral generating functions involving the Erkuş-Srivastava polynomials. The aim of this present paper is to obtain some bilateral generating functions involving the Erkuş-Srivastava polynomials and three general classes of multivariable polynomials introduced earlier by Srivastava in [A contour integral involving Fox's H-function, Indian J. Math. 14 (1972), pp. 1-6], [A multilinear generating function for the Konhauser sets of biorthogonal polynomials suggested by the Laguerre polynomials, Pacific J. Math. 117 (1985), pp. 183-191] and by Kaanoğlu and Özarslan in [Two-sided generating functions for certain class of r-variable polynomials, Mathematical and Computer Modelling 54 (2011), pp. 625-631]. Special cases involving the (Srivastava-Daoust) generalized Lauricella functions are also given.
\end{abstract}

\section{Introduction}

The Chan-Chyan-Srivastava polynomials $g_{n}^{\left(\alpha_{1}, \ldots, \alpha_{r}\right)}\left(x_{1}, \ldots, x_{r}\right)$ are a multivariable extension of the Lagrange polynomials generated by the following relation [2, p. 140, Eq. (4)]:

$$
\prod_{j=1}^{r}\left(1-x_{j} z\right)^{-\alpha_{j}}=\sum_{n=0}^{\infty} g_{n}^{\left(\alpha_{1}, \ldots, \alpha_{r}\right)}\left(x_{1}, \ldots, x_{r}\right) z^{n} \quad\left(|z|<\min \left\{\left|x_{1}\right|^{-1}, \ldots,\left|x_{r}\right|^{-1}\right\}\right) .
$$

Obviously, setting $r=2, \alpha_{1}=\alpha$ and $\alpha_{2}=\beta$ in the last equation yields the familiar Lagrange polynomials $g_{n}^{(\alpha, \beta)}\left(x_{1}, x_{2}\right)$ which occur in some statistical problems [5, p. 267]. The last generating function (1.1) yields the explicit representation [2, p. 140, Eq. (6)]:

$$
g_{n}^{\left(\alpha_{1}, \ldots, \alpha_{r}\right)}\left(x_{1}, \ldots, x_{r}\right)=\sum_{k_{1}+\cdots+k_{r}=n}\left(\alpha_{1}\right)_{k_{1}} \cdots\left(\alpha_{r}\right)_{k_{r}} \frac{x_{1}^{k_{1}}}{k_{1} !} \cdots \frac{x_{r}^{k_{r}}}{k_{r} !}
$$

Received October 31, 2012, accepted Febuary 27, 2014.

Key words and phrases. Chan-Chyan-Srivastava polynomials, Erkuş-Srivastava polynomials, Srivastava polynomials, (Srivastava-Daoust) generalized Lauricella functions, bilateral generating functions, special functions.

Corresponding author: S. Gaboury. 
or, equivalently, [12, p. 522, Eq. (17)]

$$
g_{n}^{\left(\alpha_{1}, \ldots, \alpha_{r}\right)}\left(x_{1}, \ldots, x_{r}\right)=\sum_{n_{r-1}=0}^{n} \sum_{n_{r-2}=0}^{n_{r-1}} \cdots \sum_{n_{1}=0}^{n_{2}} \frac{\left(\alpha_{1}\right)_{n_{1}}\left(\alpha_{2}\right)_{n_{2}-n_{1}} \cdots\left(\alpha_{r}\right)_{n-n_{r-1}}}{n_{1} !\left(n_{2}-n_{1}\right) ! \cdots\left(n-n_{r-1}\right) !} x_{1}^{n_{1}} x_{2}^{n_{2}-n_{1}} \cdots x_{r}^{n-n_{r-1}}
$$

where $(\lambda)_{n}$ denote the Pochhammer's symbol defined by

$$
(\lambda)_{n}:=\frac{\Gamma(\lambda+n)}{\Gamma(\lambda)} ; \quad(\lambda)_{0}=1
$$

These polynomials have been extensively investigated first by the work of Chan et al. [2] and subsequently by the works of $[3,6,7]$.

Recently, Altin and Erkuş [1, p. 239, Eq. (2)] presented a multivariable extension of the Lagrange-Hermite polynomials. This extension is given by the following generating function:

$$
\begin{gathered}
\prod_{j=1}^{r}\left(1-x_{j} z^{j}\right)^{-\alpha_{j}}=\sum_{n=0}^{\infty} h_{n}^{\left(\alpha_{1}, \ldots, \alpha_{r}\right)}\left(x_{1}, \ldots, x_{r}\right) z^{n} \\
\left(\alpha_{j} \in \mathbb{C}(j=1, \ldots, r) ;|z|<\min \left\{\left|x_{1}\right|^{-1},\left|x_{2}\right|^{-1 / 2}, \ldots,\left|x_{r}\right|^{-1 / r}\right\}\right) .
\end{gathered}
$$

Setting $r=2$ yields the well-known two variables Lagrange-Hermite polynomials studied by Dattoli et al. [4].

Shortly after, Erkuş and Srivastava proposed a new class of multivariable polynomials $\mathscr{U}_{n ; l_{1}, \ldots, l_{r}}^{\left(\alpha_{1}, \ldots, \alpha_{r}\right)}\left(x_{1}, \ldots, x_{r}\right)$ which are defined by [7, p. 268, Eq. (3)]:

$$
\begin{gathered}
\prod_{j=1}^{r}\left(1-x_{j} z^{l_{j}}\right)^{-\alpha_{j}}=\sum_{n=0}^{\infty} \mathscr{U}_{n ; l_{1}, \ldots, l_{r}}^{\left(\alpha_{1}, \ldots, \alpha_{r}\right)}\left(x_{1}, \ldots, x_{r}\right) z^{n} \\
\left(\alpha_{j} \in \mathbb{C}(j=1, \ldots, r) ; l_{j} \in \mathbb{N}(j=1, \ldots, r) ;|z|<\min \left\{\left|x_{1}\right|^{-1 / l_{1}}, \ldots,\left|x_{r}\right|^{-1 / l_{r}}\right\}\right) .
\end{gathered}
$$

This family of polynomials is a generalization and a unification of several known families of multivariable polynomials including the Chan-Chyan-Srivastava polynomials given by (1.1) and the Lagrange-Hermite polynomials defined by (1.4). Obviously, setting

$$
l_{j}=1(j=1, \ldots, r)
$$

we have

$$
\mathscr{U}_{n ; 1, \ldots, 1}^{\left(\alpha_{1}, \ldots, \alpha_{r}\right)}\left(x_{1}, \ldots, x_{r}\right)=g_{n}^{\left(\alpha_{1}, \ldots, \alpha_{r}\right)}\left(x_{1}, \ldots, x_{r}\right) .
$$

Moreover, the Lagrange-Hermite polynomials follow by substituting

$$
l_{j}=j(j=1, \ldots, r)
$$


We thus have

$$
\mathscr{U}_{n ; 1,2, \ldots, r}^{\left(\alpha_{1}, \ldots, \alpha_{r}\right)}\left(x_{1}, \ldots, x_{r}\right)=h_{n}^{\left(\alpha_{1}, \ldots, \alpha_{r}\right)}\left(x_{1}, \ldots, x_{r}\right) .
$$

The multivariable Erkuş-Srivastava polynomials have the following explicit representation [7, p. 268, Eq. (4)]:

$$
\mathscr{U}_{n ; l_{1}, \ldots, l_{r}}^{\left(\alpha_{1}, \ldots, \alpha_{r}\right)}\left(x_{1}, \ldots, x_{r}\right)=\sum_{l_{1} k_{1}+\cdots+l_{r} k_{r}=n}\left(\alpha_{1}\right)_{k_{1}} \cdots\left(\alpha_{r}\right)_{k_{r}} \frac{x_{1}^{k_{1}}}{k_{1} !} \cdots \frac{x_{r}^{k_{r}}}{k_{r} !}
$$

which gives as a special case (1.2) when

$$
l_{j}=1(j=1, \ldots, r)
$$

Almost four decades ago, Srivastava [15, p. 1, Eq. (1)] introduced and investigated the general class of polynomials $S_{n}^{m}(x)$ defined by

$$
S_{n}^{m}(x)=\sum_{k=0}^{\left[\frac{n}{m}\right]} \frac{(-n)_{m k}}{k !} A_{n, k} x^{k} \quad(n=0,1,2, \ldots),
$$

where $m$ is an arbitrary positive integer, the coefficients $A_{n, k}(n, k \geq 0)$ are arbitrary real or complex constants and $[x]$ denotes the largest integer not greater than $x$. By suitably specializing the coefficients $A_{n, k}$, the polynomials $S_{n}^{m}(x)$ can be reduced to the classical orthogonal polynomials (Jacobi polynomials, Hermite polynomials, Laguerre polynomials, see for details $\left[15,20\right.$ ? ]). Other interesting special cases of the polynomials $S_{n}^{m}(x)$ include the generalized hypergeometric polynomials such as the Bessel polynomials $y_{n}(x, \alpha, \beta)$ investigated by Krall and Frink [11, p. 108, Eq. (34)] and the generalized Hermite polynomials $g_{n}^{m}(x, h)$ considered by Gould and Hopper [9, p. 58].

In 1987, Srivastava and Garg [17, p. 686, Eq. (1.4)] introduced the multivariable analogue of the polynomials $S_{n}^{m}(x)$. This new class of polynomials $S_{n}^{m_{1}, \ldots, m_{s}}\left(x_{1}, \ldots, x_{s}\right)$ is defined by

$$
S_{n}^{m_{1}, \ldots, m_{s}}\left(x_{1}, \ldots, x_{s}\right)=\sum_{k_{1}, \ldots, k_{s}=0}^{m_{1} k_{1}+\cdots+m_{s} k_{s} \leq n}(-n)_{m_{1} k_{1}+\cdots+m_{s} k_{s}} \Lambda\left(n ; k_{1}, \ldots, k_{s}\right) \frac{x_{1}^{k_{1}} \cdots x_{s}^{k_{s}}}{k_{1} ! \cdots k_{s} !}
$$

where $m_{1}, \ldots, m_{s}$ are arbitrary positive integers, and the coefficients

$$
\Lambda\left(n ; k_{1}, \ldots, k_{s}\right) \quad\left(n, k_{i} \geq 0, i=1, \ldots, s\right)
$$

are arbitrary real or complex constants.

Another interesting class of generalized multivariable polynomials, namely the polynomials $S_{n_{1}, \ldots, n_{s}}^{m_{1}, \ldots, m_{s}}\left(x_{1}, \ldots, x_{s}\right)$ has been given in 1985 by Srivastava [16, p. 185, Eq. (7)]. These polynomials are defined as follows:

$$
S_{n_{1}, \ldots, n_{s}}^{m_{1}, \ldots, m_{s}}\left(x_{1}, \ldots, x_{s}\right)=\sum_{k_{1}=0}^{\left[\frac{n_{1}}{m_{1}}\right]} \cdots \sum_{k_{s}=0}^{\left[\frac{n_{s}}{m_{s}}\right]} \frac{\left(-n_{1}\right)_{m_{1} k_{1}} \cdots\left(-n_{s}\right)_{m_{s} k_{s}}}{k_{1} ! \cdots k_{s} !} \Omega\left(n ; k_{1}, \ldots, k_{s}\right) x_{1}^{k_{1}} \cdots x_{s}^{k_{s}}
$$


where $m_{1}, \ldots, m_{s}$ are arbitrary positive integers, $n_{1}, \ldots, n_{s}$ are arbitrary non negative integers and the coefficients

$$
\Omega\left(n ; k_{1}, \ldots, k_{s}\right) \quad\left(n, k_{i} \geq 0, i=1, \ldots, s\right)
$$

are arbitrary real or complex constants.

These classes of polynomials are related to the Srivastava-Daoust generalized Lauricella function [18, p.37 et seq.] defined as follows:

$$
\begin{aligned}
& F_{C: D^{(1)}, \ldots, D^{(s)}}^{A: B^{(1)}, \ldots B^{(s)}}\left[\begin{array}{cc}
{\left[(a): \theta^{(1)}, \ldots, \theta^{(s)}\right]:\left[b^{(1)} ; \phi^{(1)}\right] ;} & \ldots ;\left[b^{(s)} ; \phi^{(s)}\right] ; \\
{\left[(c): \psi^{(1)}, \ldots, \psi^{(s)}\right]:\left[d^{(1)} ; \delta^{(1)}\right] ;} & \ldots ;\left[d^{(s)} ; \delta^{(s)}\right] ;
\end{array}\right] \\
& \quad=\sum_{m_{1}, \ldots, m_{s}=0}^{\infty} \Delta\left(m_{1}, \ldots, m_{s}\right) \frac{z_{1}^{m_{1}}}{m_{1} !} \ldots \frac{z_{s}^{m_{s}}}{m_{s} !}
\end{aligned}
$$

where, for convenience,

$$
\Delta\left(m_{1}, \ldots, m_{s}\right):=\frac{\prod_{j=1}^{A}\left(a_{j}\right)_{m_{1}} \theta_{j}^{(1)}+\cdots+m_{s} \theta_{j}^{(s)} \prod_{j=1}^{B^{(1)}}\left(b_{j}^{(1)}\right)_{m_{1} \phi_{j}^{(1)}} \cdots \prod_{j=1}^{B^{(s)}}\left(b_{j}^{(s)}\right)_{m_{s} \phi_{j}^{(s)}}}{\prod_{j=1}^{C}\left(c_{j}\right)_{m_{1}} \psi_{j}^{(1)}+\cdots+m_{s} \psi_{j}^{(s)} \prod_{j=1}^{D^{(1)}}\left(d_{j}^{(1)}\right)_{m_{1}} \delta_{j}^{(1)} \cdots \prod_{j=1}^{D^{(s)}}\left(d_{j}^{(s)}\right)_{m_{s}} \delta_{j}^{(s)}} .
$$

The coefficients

$$
\begin{aligned}
& \theta_{j}^{(k)}(j=1, \ldots, A ; k=1, \ldots, s), \quad \phi_{j}^{(k)}\left(j=1, \ldots, B^{(k)} ; k=1, \ldots, s\right), \\
& \psi_{j}^{(k)}(j=1, \ldots, C ; k=1, \ldots, s) \quad \text { and } \quad \delta_{j}^{(k)}\left(j=1, \ldots, D^{(k)} ; k=1, \ldots, s\right)
\end{aligned}
$$

are real constants and $\left(b_{B^{(k)}}^{(k)}\right)$ abbreviates the array of $B^{(k)}$ parameters

$$
b_{j}^{(k)} \quad\left(j=1, \ldots, B^{(k)} ; k=1, \ldots, s\right)
$$

with similar interpretations for other sets of parameters.

By assigning suitably special values to the arbitrary coefficient $\Lambda\left(n ; k_{1}, \ldots, k_{s}\right)$ and $\Omega\left(n ; k_{1}, \ldots, k_{s}\right)$ of equations (1.8) and (1.9) respectively, we arrive to the following special cases.

Setting

$$
\Lambda\left(n ; k_{1}, \ldots, k_{s}\right)=\Omega\left(n ; k_{1}, \ldots, k_{s}\right)=\frac{\prod_{j=1}^{A}\left(a_{j}\right)_{m_{1}} \theta_{j}^{(1)}+\cdots+m_{s} \theta_{j}^{(s)} \prod_{j=1}^{B^{(1)}}\left(b_{j}^{(1)}\right)_{m_{1} \phi_{j}^{(1)}} \cdots \prod_{j=1}^{B^{(s)}}\left(b_{j}^{(s)}\right)_{m_{s}} \phi_{j}^{(s)}}{\prod_{j=1}^{C}\left(c_{j}\right)_{m_{1}} \psi_{j}^{(1)}+\cdots+m_{s} \psi_{j}^{(s)} \prod_{j=1}^{D^{(1)}}\left(d_{j}^{(1)}\right)_{m_{1}} \delta_{j}^{(1)} \cdots \prod_{j=1}^{D^{(s)}}\left(d_{j}^{(s)}\right)_{m_{s} \delta_{j}^{(s)}}}
$$

in (1.8) and (1.9), we obtain respectively

$$
S_{n}^{m_{1}, \ldots, m_{s}}\left(x_{1}, \ldots, x_{s}\right)=F_{C: D^{(1)} ; \ldots, D^{(s)}}^{A+1 ; B^{(1)}}
$$




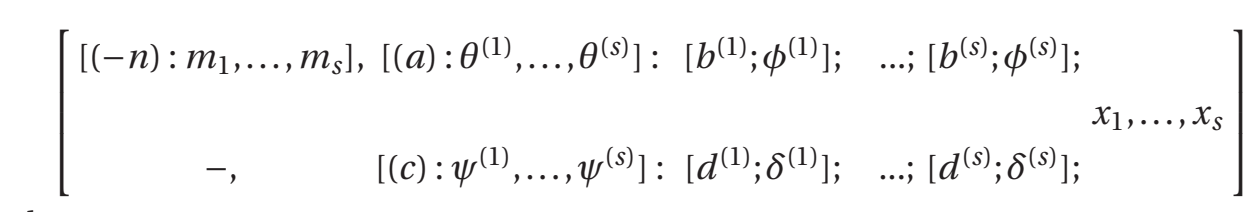

and

$$
\begin{aligned}
& S_{n_{1}, \ldots, n_{s}}^{m_{1}, \ldots, m_{s}}\left(x_{1}, \ldots, x_{s}\right)=F_{C: D^{(1)} ; \ldots, D^{(s)}}^{A: 1+B^{(1)} ; \ldots ; 1+B^{(s)}} \\
& {\left[\begin{array}{cccc}
{\left[(a): \theta^{(1)}, \ldots, \theta^{(s)}\right]:\left[-n_{1} ; m_{1}\right],\left[b^{(1)} ; \phi^{(1)}\right] ;} & \ldots ; & {\left[-n_{s} ; m_{s}\right],\left[b^{(s)} ; \phi^{(s)}\right] ;} \\
{\left[(c): \psi^{(1)}, \ldots, \psi^{(s)}\right]:} & -, \quad\left[d^{(1)} ; \delta^{(1)}\right] ; & \ldots ; & -, \quad\left[d^{(s)} ; \delta^{(s)}\right] ;
\end{array}\right] .}
\end{aligned}
$$

Finally, in 2011, Kaanoğlu and Özarslan [10] introduced a certain class of multivariable polynomials $P_{n}^{m, N_{1}, \ldots, N_{r-1}}\left(x_{1}, \ldots, x_{r}\right)$ and obtained two-sided linear generating functions for this class of polynomials. These polynomials are a generalization of the three variables polynomials studied by Srivastava et al. [19]. Explicitly, the polynomials $P_{n}^{m, N_{1}, \ldots, N_{r-1}}\left(x_{1}, \ldots, x_{r}\right)$ are defined by

$$
\begin{gathered}
P_{n}^{m, N_{1}, \ldots, N_{r-1}}\left(x_{1}, \ldots, x_{r}\right)=\sum_{k_{r-1}=0}^{\left[\frac{n}{N_{r-1}}\right]} \sum_{k_{r-2}=0}^{\left[\frac{k_{r-1}}{N_{r-2}}\right]} \cdots \sum_{k_{2}=0}^{\left[\frac{k_{3}}{N_{2}}\right]} \sum_{k_{1}=0}^{\left[\frac{k_{2}}{N_{1}}\right]} A_{m+n, k_{1}, \ldots, k_{r-1}} \frac{x_{1}^{k_{1}} x_{2}^{k_{2}-N_{1} k_{1}} \cdots x_{r}^{n-N_{r-1} k_{r-1}}}{k_{1} !\left(k_{2}-N_{1} k_{1}\right) ! \cdots\left(n-N_{r-1} k_{r-1}\right) !} \\
\left(m, n \in \mathbb{N}_{0} ; N_{1}, N_{2}, \ldots, N_{r-1} \in \mathbb{N}\right)
\end{gathered}
$$

where $\left\{A_{m+n, k_{1}, \ldots, k_{r-1}}\right\}$ is a sequence of complex numbers.

Following the works of Liu et al. [12, 13, 14], we propose, in this paper, some bilateral generating functions involving the Erkuş-Srivastava polynomials and the three classes of generalized polynomials defined above in (1.8), (1.9) and (1.14). Some special cases are computed and presented under the form of corollaries.

\section{Main results}

In this section, we present some bilateral generating functions involving the Erkuş-Srivastava polynomials and the three classes of polynomials respectively defined previously by (1.8), (1.9) and (1.14). Some corollaries are also given as examples of applications of these presumably new generating functions.

We begin this section by deriving a relationship between the Erkuş-Srivastava polynomials and the Chan-Chyan-Srivastava polynomials. Considering the generating functions (1.1) and (1.5), we find the following relation:

$$
\sum_{n=0}^{\infty} \mathscr{U}_{n ; l_{1}, \ldots, l_{r}}^{\left(\alpha_{1}, \ldots, \alpha_{r}\right)}\left(x_{1}, \ldots, x_{r}\right) z^{n}=\prod_{i=1}^{r}\left(1-x_{i} z^{l_{i}}\right)^{-\alpha_{i}}=\prod_{i=1}^{r} \prod_{j=1}^{l_{i}}\left(1-\omega_{i j} z\right)^{-\alpha_{i}}
$$




$$
=\sum_{n=0}^{\infty} g_{n}^{\left(\alpha_{1}, \ldots, \alpha_{1}, \ldots, \alpha_{r}, \ldots, \alpha_{r}\right)}\left(\omega_{11}, \ldots, \omega_{1 l_{1}}, \ldots, \omega_{r 1}, \ldots, \omega_{r l_{r}}\right) z^{n}
$$

where we have implicitly assumed that the set:

$$
\left\{\omega_{i j}: 1 \leq i \leq r \text { and } 1 \leq j \leq l_{i}\left(l_{i} \in \mathbb{N}: i=1, \ldots, r\right)\right\}
$$

which depends upon the $l_{i}$ distinct values of the factor $x_{i}^{\frac{1}{l_{i}}}$ occurring in the expression:

$$
1-\left(x_{i}^{\frac{1}{l_{i}}} z\right)^{l_{i}} \quad(i=1, \ldots, r)
$$

exists such that

$$
\left(1-x_{i} z^{l_{i}}\right)^{-\alpha_{i}}=\prod_{j=1}^{l_{i}}\left(1-\omega_{i j} z\right)^{-\alpha_{i}} \quad(i=1, \ldots, r) .
$$

Thus, the following relationship holds:

$$
\mathscr{U}_{n ; l_{1}, \ldots, l_{r}}^{\left(\alpha_{1}, \ldots, \alpha_{r}\right)}\left(x_{1}, \ldots, x_{r}\right)=g_{n}^{\left(\alpha_{1}, \ldots, \alpha_{1}, \ldots, \alpha_{r}, \ldots, \alpha_{r}\right)}\left(\omega_{11}, \ldots, \omega_{1 l_{1}}, \ldots, \omega_{r 1}, \ldots, \omega_{r l_{r}}\right)
$$

The following lemma, given in [12, p. 521, Eq. (13)], will be useful in the sequel.

\section{Lemma 2.1. The following multiple summation formula}

$$
\sum_{n_{r}=0}^{\infty} \sum_{n_{r-1}=0}^{n_{r}} \ldots \sum_{n_{1}=0}^{n_{2}} A\left(n_{1}, n_{2}, \ldots, n_{r}\right)=\sum_{n_{r}=0}^{\infty} \sum_{n_{r-1}=0}^{\infty} \ldots \sum_{n_{1}=0}^{\infty} A\left(n_{1}, n_{1}+n_{2}, \ldots, n_{1}+n_{2}+\cdots+n_{r}\right)
$$

holds true provided that each of the series involved is absolutely convergent.

For a suitably bounded non-vanishing multiple sequence $\left\{\Omega\left(k_{1}, \ldots, k_{s}\right)\right\}_{k_{1}, \ldots, k_{s} \in \mathbb{N}_{0}}$ of real or complex parameters, we define a function $\Phi_{n}\left(n_{2}, m_{2} ; \ldots ; n_{s}, m_{s} ; y_{1}, \ldots, y_{s}\right)$ of $s$ variables where $m_{j} \in \mathbb{N}$, for $j=2, \ldots, s$, and $n_{j} \in \mathbb{N}_{0}$, for $j=2, \ldots, s$, by

$$
\begin{aligned}
\Phi_{n}\left(n_{2}, m_{2} ; \ldots ; n_{s}, m_{s} ; y_{1}, \ldots, y_{s}\right)=S_{n, n_{2}, \ldots, n_{s}}^{1, m_{2}, \ldots, m_{s}}\left(y_{1}, \ldots, y_{s}\right) \\
\quad=\sum_{k_{1}=0}^{n} \sum_{k_{2}=0}^{\left[\frac{n_{2}}{m_{2}}\right]} \cdots \sum_{k_{S}=0}^{\left[\frac{n_{s}}{m_{S}}\right]} \frac{(-n)_{k_{1}}\left(-n_{2}\right)_{m_{2} k_{2}} \cdots\left(-n_{s}\right)_{m_{s} k_{s}}}{k_{1} ! \cdots k_{s} !} \Omega\left(k_{1}, \ldots, k_{s}\right) y_{1}^{k_{1}} \cdots y_{s}^{k_{s}}
\end{aligned}
$$

where $S_{n, n_{2}, \ldots, n_{s}}^{1, m_{2}, \ldots, m_{s}}\left(y_{1}, \ldots, y_{s}\right)$ denotes the generalized Srivastava polynomials defined by (1.9). As usual, $[x]$ denotes the greatest integer in $x$ and

$$
\mathbb{N}_{0}:=\mathbb{N} \cup\{0\}
$$


Theorem 2.2. The following bilateral generating function holds true:

$$
\begin{aligned}
\sum_{n=0}^{\infty} \mathcal{U}_{n ; l_{1}, \ldots, l_{r}}^{\left(\alpha_{1}, \ldots, \alpha_{r}\right)}\left(x_{1}, \ldots, x_{r}\right) S_{n, n_{2}, \ldots, n_{s}}^{1, m_{2}, \ldots, m_{s}}\left(y_{1}, \ldots, y_{s}\right) z^{n} \\
=\prod_{i=1}^{r} \prod_{j=1}^{l_{i}}\left(1-\omega_{i j} z\right)^{-\alpha_{i}} \sum_{\left(t_{1}\right), \ldots,\left(t_{r}\right)=0}^{\infty} \sum_{k_{2}=0}^{\left[\frac{n_{2}}{m_{2}}\right]} \cdots \sum_{k_{s}=0}^{\left[\frac{n_{S}}{m_{S}}\right]} \frac{\left(-n_{2}\right)_{m_{2} k_{2}} \cdots\left(-n_{s}\right)_{m_{s} k_{s}}}{k_{2} ! \cdots k_{s} !} \\
\quad \times \Omega\left(\Re, k_{2}, \ldots, k_{s}\right) \cdot\left(\prod_{i=1}^{r} \prod_{j=1}^{l_{i}} \frac{\left(\alpha_{i}\right)_{t_{i j}}}{t_{i j} !}\left(\frac{\omega_{i j} y_{1} z}{\omega_{i j} z-1}\right)^{t_{i j}}\right) y_{2}^{k_{2}} \cdots y_{s}^{k_{s}},
\end{aligned}
$$

where

$$
\left(t_{i}\right):=t_{i 1}, \ldots, t_{i l_{i}}(i=1, \ldots, r) \text { and } \Re:=\sum_{i=1}^{r} \sum_{j=1}^{l_{i}} t_{i j}
$$

Proof. It is easy to see that

$$
\begin{aligned}
& \sum_{n=0}^{\infty} \mathscr{U}_{n ; l_{1}, \ldots, l_{r}}^{\left(\alpha_{1}, \ldots, \alpha_{r}\right)}\left(x_{1}, \ldots, x_{r}\right) S_{n, n_{2}, \ldots, n_{s}}^{1, m_{2}, \ldots, m_{s}}\left(y_{1}, \ldots, y_{s}\right) z^{n} \\
& =\sum_{n=0}^{\infty} \mathscr{U}_{n ; l_{1}, \ldots, l_{r}}^{\left(\alpha_{1}, \ldots, \alpha_{r}\right)}\left(x_{1}, \ldots, x_{r}\right) \sum_{k_{1}=0}^{n} \sum_{k_{2}=0}^{\left[\frac{n_{2}}{m_{2}}\right]} \cdots \sum_{k_{S}=0}^{\left[\frac{n_{S}}{m_{S}}\right]} \frac{(-n)_{k_{1}}\left(-n_{2}\right)_{m_{2} k_{2}} \cdots\left(-n_{s}\right)_{m_{s} k_{s}}}{k_{1} ! \cdots k_{s} !} \\
& \times \Omega\left(k_{1}, \ldots, k_{s}\right) y_{1}^{k_{1}} \cdots y_{s}^{k_{s}} z^{n} \\
& =\sum_{n=0}^{\infty} \sum_{k_{1}=0}^{\infty} \sum_{k_{2}=0}^{\left[\frac{n_{2}}{m_{2}}\right]} \ldots \sum_{k_{S}=0}^{\left[\frac{n_{S}}{m_{S}}\right]}\left(\begin{array}{c}
n+k_{1} \\
n
\end{array}\right) g_{n+k_{1}}^{\left(\alpha_{1}, \ldots, \alpha_{1}, \ldots, \alpha_{r}, \ldots, \alpha_{r}\right)}\left(\omega_{11}, \ldots, \omega_{1 l_{1}}, \ldots, \omega_{r 1}, \ldots, \omega_{r l_{r}}\right) \\
& \times \frac{\left(-n_{2}\right)_{m_{2} k_{2}} \cdots\left(-n_{s}\right)_{m_{s}} k_{s}}{k_{2} ! \cdots k_{s} !} \Omega\left(k_{1}, \ldots, k_{s}\right)\left(-y_{1} z\right)^{k_{1}} \cdots y_{s}^{k_{s}} z^{n} .
\end{aligned}
$$

Now, by making use of the following formula [2, p. 143, Eq. (20)]:

$$
\sum_{n=0}^{\infty}\left(\begin{array}{c}
n+m \\
n
\end{array}\right) g_{n+m}^{\left(\alpha_{1}, \ldots, \alpha_{r}\right)}\left(x_{1}, \ldots, x_{r}\right) z^{n}=\prod_{j=1}^{r}\left(1-x_{j} z\right)^{-\alpha_{j}} g_{m}^{\left(\alpha_{1}, \ldots, \alpha_{r}\right)}\left(\frac{x_{1}}{1-x_{1} z}, \ldots, \frac{x_{r}}{1-x_{r} z}\right) \quad\left(m \in \mathbb{N}_{0}\right)
$$

and equation (1.3), we obtain

$$
\begin{aligned}
& \sum_{n=0}^{\infty} \mathscr{U}_{n ; l_{1}, \ldots, l_{r}}^{\left(\alpha_{1}, \ldots, \alpha_{r}\right)}\left(x_{1}, \ldots, x_{r}\right) S_{n, n_{2}, \ldots, n_{s}}^{1, m_{2}, \ldots, m_{s}}\left(y_{1}, \ldots, y_{s}\right) z^{n} \\
& =\sum_{k_{1}=0}^{\infty} \sum_{k_{2}=0}^{\left[\frac{n_{2}}{m_{2}}\right]} \cdots \sum_{k_{S}=0}^{\left[\frac{n_{S}}{m_{S}}\right]} \frac{\left(-n_{2}\right)_{m_{2} k_{2}} \cdots\left(-n_{s}\right)_{m_{s}} k_{s}}{k_{2} ! \cdots k_{s} !} \Omega\left(k_{1}, \ldots, k_{s}\right)\left(-y_{1} z\right)^{k_{1}} \cdots y_{s}^{k_{s}} \\
& \quad \times \prod_{i=1}^{r} \prod_{j=1}^{l_{i}}\left(1-\omega_{i j} z\right)^{-\alpha_{i}}
\end{aligned}
$$




$$
\times g_{k_{1}}^{\left(\alpha_{1}, \ldots, \alpha_{1}, \ldots, \alpha_{r}, \ldots, \alpha_{r}\right)}\left(\frac{\omega_{11}}{1-\omega_{11} z}, \ldots, \frac{\omega_{1 l_{1}}}{1-\omega_{1 l_{1}} z}, \ldots, \frac{\omega_{r 1}}{1-\omega_{r 1} z}, \ldots, \frac{\omega_{r l_{r}}}{1-\omega_{r l_{r}} z}\right) .
$$

Using (1.3) again yields

$$
\begin{aligned}
& \sum_{n=0}^{\infty} \mathscr{U}_{n ; l_{1}, \ldots, l_{r}}^{\left(\alpha_{1}, \ldots, \alpha_{r}\right)}\left(x_{1}, \ldots, x_{r}\right) S_{n, n_{2}, \ldots, n_{s}}^{1, m_{2}, \ldots, m_{s}}\left(y_{1}, \ldots, y_{s}\right) z^{n} \\
& =\sum_{k_{1}=0}^{\infty} \sum_{k_{2}=0}^{\left[\frac{n_{2}}{m_{2}}\right]} \cdots \sum_{k_{S}=0}^{\left[\frac{n_{S}}{m_{S}}\right]} \frac{\left(-n_{2}\right)_{m_{2} k_{2}} \cdots\left(-n_{s}\right)_{m_{s}} k_{s}}{k_{2} ! \cdots k_{s} !} \Omega\left(k_{1}, \ldots, k_{s}\right)\left(-y_{1} z\right)^{k_{1}} \cdots y_{s}^{k_{s}} \\
& \times \prod_{i=1}^{r} \prod_{j=1}^{l_{i}}\left(1-\omega_{i j} z\right)^{-\alpha_{i}} \sum_{t_{r, l} l_{r}-1}^{k_{1}} \sum_{t_{r, l_{r}-2}=0}^{t_{r, l_{r}-1}} \ldots \sum_{t_{r, l_{r-1}-1}=0}^{t_{r, 1}} \ldots \sum_{t_{r-1, l_{r}-2}=0}^{t_{r-1, l_{r}-1}} \ldots \sum_{t_{1,1}=0}^{t_{1,2}} \\
& \times \frac{\left(\alpha_{1}\right)_{t_{1,1}}\left(\alpha_{1}\right)_{t_{1,2}-t_{1,1}} \cdots\left(\alpha_{1}\right)_{t_{1, l_{1}}-t_{1, l_{1}-1}}\left(\alpha_{2}\right)_{t_{2,1}-t_{1, l_{1}}}\left(\alpha_{2}\right)_{t_{2,2}-t_{2,1}} \cdots\left(\alpha_{2}\right)_{t_{2, l_{2}}-t_{2, l_{2}-1}}}{t_{1,1} !\left(t_{1,2}-t_{1,1}\right) ! \cdots\left(t_{1, l_{1}}-t_{1, l_{1}-1}\right) !\left(t_{2,1}-t_{1, l_{1}}\right) !\left(t_{2,2}-t_{2,1}\right) ! \cdots\left(t_{2, l_{2}}-t_{1, l_{1}-1}\right) !} \\
& \times \frac{\cdots\left(\alpha_{r}\right)_{t_{r, 1}-t_{r-1, l_{r-1}}}\left(\alpha_{r}\right)_{t_{r, 2}-t_{r, 1}} \cdots\left(\alpha_{r}\right)_{k_{1}-t_{r, l_{r}-1}}}{\cdots\left(t_{r, 1}-t_{r-1, l_{r-1}}\right) !\left(t_{r, 2}-t_{r, 1}\right) ! \cdots\left(k_{1}-t_{r, l_{r}-1}\right) !}\left(\frac{\omega_{11}}{1-\omega_{11} z}\right)^{t_{1,1}} \\
& \times\left(\frac{\omega_{12}}{1-\omega_{12} z}\right)^{t_{1,2}-t_{1,1}} \cdots\left(\frac{\omega_{1 l_{1}}}{1-\omega_{1 l_{1}} z}\right)^{t_{1, l_{1}}-t_{1, l_{1}-1}} \cdots\left(\frac{\omega_{r 1}}{1-\omega_{r 1} z}\right)^{t_{r, 1}-t_{r-1, l_{r-1}}} \\
& \times\left(\frac{\omega_{r 2}}{1-\omega_{r 2} z}\right)^{t_{r, 2}-t_{r, 1}} \cdots\left(\frac{\omega_{r l_{r}}}{1-\omega_{r l_{r}} z}\right)^{k_{1}-t_{r, l_{r}-1}} \text {. }
\end{aligned}
$$

Applying Lemma 2.1 to (2.10) gives the desired result after simple manipulations.

Corollary 2.3. In view of equations (1.13) and (2.6), we have the following relation

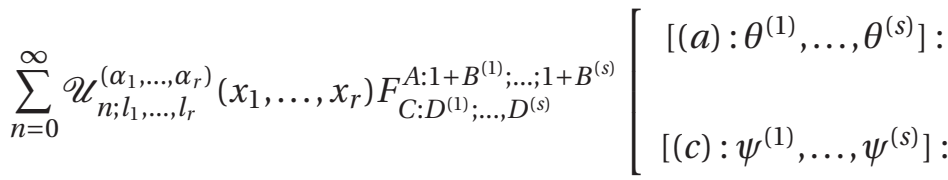

$$
\begin{aligned}
& \begin{array}{c}
{[-n ; 1],\left[b^{(1)} ; \phi^{(1)}\right] ; \quad \ldots ;\left[-n_{s} ; m_{s}\right],\left[b^{(s)} ; \phi^{(s)}\right] ;} \\
-, \quad\left[d^{(1)} ; \delta^{(1)}\right] ; \quad \ldots ; \quad-, \quad\left[d^{(s)} ; \delta^{(s)}\right] ;
\end{array} \\
& =\prod_{i=1}^{r} \prod_{j=1}^{l_{i}}\left(1-\omega_{i j} z\right)^{-\alpha_{i}} F_{C+D: 0 ; \ldots ; 0 ; D^{(2)} ; \ldots, D^{(s)}}^{A+B: 1 ; ; 1 ; 1+B^{(2)}, \ldots ; 1+B^{(s)}} \\
& {\left[(e): \varphi^{(1)}, \ldots, \varphi^{\left(l_{1}+\cdots+l_{r}+s-1\right)}\right]:\left[\alpha_{1} ; 1\right] ; \ldots ;\left[\alpha_{1} ; 1\right] ; \ldots ;\left[\alpha_{r} ; 1\right] ; \ldots ;\left[\alpha_{r} ; 1\right] ;} \\
& {\left[(f): \Theta^{(1)}, \ldots, \Theta^{\left(l_{1}+\cdots+l_{r}+s-1\right)}\right]: \quad-; \quad \ldots ; \quad-; \quad \ldots ; \quad-; \quad \ldots ; \quad-;} \\
& {\left[-n_{2} ; m_{2}\right],\left[b^{(2)} ; \phi^{(2)}\right] ; \quad \ldots ;\left[-n_{s} ; m_{s}\right],\left[b^{(s)} ; \phi^{(s)}\right] \text {; }} \\
& -, \quad\left[d^{(2)} ; \delta^{(2)}\right] ; \quad \ldots ; \quad-, \quad\left[d^{(s)} ; \delta^{(s)}\right] ; \\
& \frac{\omega_{11} y_{1} z}{\omega_{11} z-1}, \ldots, \frac{\omega_{1 l_{1}} y_{1} z}{\omega_{1 l_{1}} z-1}, \ldots,
\end{aligned}
$$




$$
\left.\frac{\omega_{1 l_{r} y_{1} z}}{\omega_{1 l_{r}} z-1}, \ldots, \frac{\omega_{r l_{r}} y_{1} z}{\omega_{r l_{r}} z-1}, y_{2}, \ldots, y_{s}\right]
$$

where the coefficients $e_{j}, f_{j}, \varphi_{j}^{(k)}$ and $\Theta_{j}^{(k)}$ are given by

$$
\begin{aligned}
e_{j} & = \begin{cases}a_{j} & (1 \leq j \leq A) \\
b_{j-A} & (A<j \leq A+B),\end{cases} \\
f_{j} & = \begin{cases}c_{j} & (1 \leq j \leq E) \\
d_{j-A} & (E<j \leq E+D),\end{cases} \\
\varphi_{j}^{(k)} & = \begin{cases}\theta_{j}^{(1)} & \left(1 \leq j \leq A ; 1 \leq k \leq l_{1}+\cdots+l_{r}\right) \\
\theta_{j}^{\left(k-l_{1}-\cdots-l_{r}+1\right)} & \left(1 \leq j \leq A ; l_{1}+\cdots+l_{r}<k \leq l_{1}+\cdots+l_{r}+s-1\right) \\
\phi_{j-A} & \left(A<j \leq A+B ; 1 \leq k \leq l_{1}+\cdots+l_{r}\right) \\
0 & \left(A<j \leq A+B ; l_{1}+\cdots+l_{r}<k \leq l_{1}+\cdots+l_{r}+s-1\right)\end{cases}
\end{aligned}
$$

and

$$
\Theta_{j}^{(k)}= \begin{cases}\Psi_{j}^{(1)} & \left(1 \leq j \leq E ; 1 \leq k \leq l_{1}+\cdots+l_{r}\right) \\ \Psi_{j}^{\left(k-l_{1}-\cdots-l_{r}+1\right)} & \left(1 \leq j \leq E ; l_{1}+\cdots+l_{r}<k \leq l_{1}+\cdots+l_{r}+s-1\right) \\ \delta_{j-A} & \left(E<j \leq E+D ; 1 \leq k \leq l_{1}+\cdots+l_{r}\right) \\ 0 & \left(E<j \leq E+D ; l_{1}+\cdots+l_{r}<k \leq l_{1}+\cdots+l_{r}+s-1\right),\end{cases}
$$

respectively.

Considering now a suitably bounded non-vanishing multiple sequence

$$
\left\{\Omega\left(n, k_{1} ; n_{2}, k_{2} ; \ldots ; n_{s}, k_{s}\right)\right\}_{k_{1}, \ldots, k_{s} \in \mathbb{N}_{0}}
$$

of real or complex parameters where $n, n_{2}, \ldots, n_{s}$ are fixed non negative integers, we define a function $\Xi_{n}\left(m_{1} ; n_{2}, m_{2} ; \ldots ; n_{s}, m_{s} ; y_{1}, \ldots, y_{s}\right)$ of $s$ variables where $m_{j} \in \mathbb{N}$, for $j=1, \ldots, s$, by

$$
\begin{aligned}
& \Xi_{n}\left(m_{1} ; n_{2}, m_{2} ; \ldots ; n_{s}, m_{s} ; y_{1}, \ldots, y_{s}\right)=\frac{S_{n, n_{2}, \ldots, n_{s}}^{m_{1}, \ldots, m_{s}}\left(y_{1}, \ldots, y_{s}\right)}{\prod_{i=1}^{r}\left\{\left(1-\alpha_{i}\right)_{n}\right\}_{i}^{l_{i}}} \\
& \quad=\sum_{k_{1}=0}^{\left[\frac{n}{m_{1}}\right]} \sum_{k_{2}=0}^{\left[\frac{n_{2}}{m_{2}}\right]} \cdots \sum_{k_{s}=0}^{\left[\frac{n_{s}}{m_{S}}\right]} \frac{(-n)_{m_{1} k_{1}} \cdots\left(-n_{s}\right)_{m_{s} k_{s}}}{k_{1} ! \cdots k_{s} !} \frac{\Omega\left(n, k_{1} ; n_{2}, k_{2} ; \ldots ; n_{s}, k_{s}\right)}{\prod_{i=1}^{r}\left\{\left(1-\alpha_{i}\right)_{n}\right\}^{l_{i}}} y_{1}^{k_{1}} \cdots y_{s}^{k_{s}}
\end{aligned}
$$

where $S_{n, n_{2}, \ldots, n_{s}}^{m_{1}, \ldots, m_{s}}\left(y_{1}, \ldots, y_{s}\right)$ denotes the generalized Srivastava polynomials defined by (1.9).

Theorem 2.4. The following bilateral generating function holds true:

$$
\sum_{n=0}^{\infty} \mathscr{U}_{n ; l_{1}, \ldots, l_{r}}^{\left(\alpha_{1}-n, \ldots, \alpha_{r}-n\right)}\left(x_{1}, \ldots, x_{r}\right) \Xi_{n}\left(m_{1} ; n_{2}, m_{2} ; \ldots ; n_{s}, m_{s} ; y_{1}, \ldots, y_{s}\right) z^{n}
$$




$$
\begin{aligned}
= & \sum_{\left(t_{1}\right), \ldots,\left(t_{r}\right)=0}^{\infty}\left(\prod_{i=1}^{r} \prod_{j=1}^{l_{i}}\left(1-\alpha_{i}\right)_{\mathfrak{R}-t_{i j}}\right)^{-1} S_{\Re, n_{2}, \ldots, n_{s}}^{m_{1}, \ldots, m_{s}}\left(y_{1}, \ldots, y_{s}\right) \\
& \times \frac{\left(-\omega_{11} z\right)^{t_{11}}}{t_{11} !} \cdots \frac{\left(-\omega_{1 l_{1}} z\right)^{t_{1 l_{1}}}}{t_{1 l_{1}} !} \cdots \frac{\left(-\omega_{r 1} z\right)^{t_{r 1}}}{t_{r 1} !} \cdots \frac{\left(-\omega_{r l_{r}} z\right)^{t_{r l}}}{t_{r l_{r}} !}
\end{aligned}
$$

where

$$
\left(t_{i}\right):=t_{i 1}, \ldots, t_{i l_{i}}(i=1, \ldots, r) \text { and } \Re:=\sum_{i=1}^{r} \sum_{j=1}^{l_{i}} t_{i j} \text {. }
$$

Proof. By using (1.3) and (2.3), we have

$$
\begin{aligned}
& \sum_{n=0}^{\infty} \mathscr{U}_{n ; l_{1}, \ldots, l_{r}}^{\left(\alpha_{1}-n, \ldots, \alpha_{r}-n\right)}\left(x_{1}, \ldots, x_{r}\right) \Xi_{n}\left(m_{1} ; n_{2}, m_{2} ; \ldots ; n_{s}, m_{s} ; y_{1}, \ldots, y_{s}\right) z^{n} \\
& =\sum_{n=0}^{\infty} g_{n}^{\left(\alpha_{1}, \ldots, \alpha_{1}, \ldots, \alpha_{r}, \ldots, \alpha_{r}\right)}\left(\omega_{11}, \ldots, \omega_{1 l_{1}}, \ldots, \omega_{r 1}, \ldots, \omega_{r l_{r}}\right) \frac{S_{n, n_{2}, \ldots, n_{s}}^{m_{1}, \ldots, m_{s}}\left(y_{1}, \ldots, y_{s}\right)}{\prod_{i=1}^{r}\left\{\left(1-\alpha_{i}\right)_{n}\right\}^{l_{i}}} z^{n} \\
& =\sum_{n=0}^{\infty} \sum_{\left.t_{r(l r}-1\right)=0}^{n} \cdots \sum_{t_{r 1}=0}^{t_{r 2}} \sum_{t_{(r-1) l_{r-1}}=0}^{t_{r 1}} \cdots \sum_{t_{1\left(l_{r-1}\right)}=0}^{t_{1 l_{1}}} \cdots \sum_{t_{12}=0}^{t_{13}} \sum_{t_{11}=0}^{t_{12}} \\
& \times \frac{\left(\alpha_{1}-n\right)_{t_{11}}\left(\alpha_{1}-n\right)_{t_{12}-t_{11}} \cdots\left(\alpha_{1}-n\right)_{t_{1 l_{1}}-t_{1\left(l_{1}-1\right)}} \cdots}{t_{11} !\left(t_{12}-t_{11}\right) ! \cdots\left(t_{1 l_{1}}-t_{1\left(l_{1}-1\right)}\right) ! \cdots} \\
& \times \frac{\left(\alpha_{r}-n\right)_{t_{r 1}-t_{(r-1) l_{r-1}}}\left(\alpha_{r}-n\right)_{t_{r 2}-t_{r 1}} \cdots\left(\alpha_{r}-n\right)_{t_{n}-t_{r(l r-1)}}}{\left(t_{r 1}-t_{(r-1) l_{r-1}}\right) !\left(t_{r 2}-t_{r 1}\right) ! \cdots\left(n-t_{r\left(l_{r}-1\right)}\right) !} \frac{S_{n, n_{2}, \ldots, n_{s}}^{m_{1}, \ldots, m_{s}}\left(y_{1}, \ldots, y_{s}\right)}{\prod_{i=1}^{r}\left\{\left(1-\alpha_{i}\right)_{n}\right\}^{l_{i}}} \\
& \times \omega_{11}^{t_{11}} \omega_{12}^{t_{12}-t_{11}} \cdots \omega_{1 l_{1}}^{t_{1 l_{1}}-t_{1\left(l_{1}-1\right)}} \cdots \omega_{r 1}^{t_{r 1}-t_{(r-1) l_{r-1}}} \omega_{r 2}^{t_{r 2}-t_{r 1}} \cdots \omega_{r l_{r}}^{n-t_{r(l-1)}} z^{n} \\
& =\sum_{n=0}^{\infty} \sum_{t_{r\left(l_{r}-1\right)}=0}^{n} \cdots \sum_{t_{r 1}=0}^{t_{r 2}} \sum_{t_{(r-1) l_{r-1}}=0}^{t_{r 1}} \cdots \sum_{t_{1\left(l_{r-1}\right)}=0}^{t_{1 l_{1}}} \cdots \sum_{t_{12}=0}^{t_{13}} \sum_{t_{11}=0}^{t_{12}} \\
& \times \frac{(-1)^{n}}{\left(1-\alpha_{1}\right)_{n-t_{11}}\left(1-\alpha_{1}\right)_{n-t_{12}+t_{11}} \cdots\left(1-\alpha_{1}\right)_{n-t_{1 l_{1}}+t_{1\left(l_{1}-1\right)}}} \cdots \\
& \times \frac{S_{n, n_{2}, \ldots, n_{s}}^{m_{1}, \ldots, m_{s}}\left(y_{1}, \ldots, y_{s}\right)}{\left(1-\alpha_{r}\right)_{n-t_{r 1}+t_{(r-1)} l_{r-1}}\left(1-\alpha_{r}\right)_{n-t_{r 2}+t_{r 1}} \cdots\left(1-\alpha_{r}\right)_{t_{r\left(l_{r}-1\right)}}} \\
& \times \frac{\omega_{11}^{t_{11}} \omega_{12}^{t_{12}-t_{11}} \cdots \omega_{1 l_{1}}^{t_{1 l_{1}}-t_{1\left(l_{1}-1\right)}} \cdots \omega_{r 1}^{t_{r 1}-t_{(r-1) l_{r-1}}} \omega_{r 2}^{t_{r 2}-t_{r 1}} \cdots \omega_{r l_{r}}^{n-t_{r\left(l_{r}-1\right)}}}{t_{11} !\left(t_{12}-t_{11}\right) ! \cdots\left(t_{1 l_{1}}-t_{1\left(l_{1}-1\right)}\right) ! \cdots\left(t_{r 1}-t_{(r-1) l_{r-1}}\right) !\left(t_{r 2}-t_{r 1}\right) ! \cdots\left(n-t_{r\left(l_{r}-1\right)}\right) !} z^{n}
\end{aligned}
$$

Applying Lemma 2.1 to the last relation, we find

$$
\begin{aligned}
& \sum_{n=0}^{\infty} \mathscr{U}_{n ; l_{1}, \ldots, l_{r}}^{\left(\alpha_{1}-n, \ldots, \alpha_{r}-n\right)}\left(x_{1}, \ldots, x_{r}\right) \Xi_{n}\left(m_{1} ; n_{2}, m_{2} ; \ldots ; n_{s}, m_{s} ; y_{1}, \ldots, y_{s}\right) z^{n} \\
& =\sum_{\left.\left(t_{1}\right), \ldots, t_{r}\right)=0}^{\infty} \frac{\omega_{11}^{t_{11}} \omega_{12}^{t_{12}} \cdots \omega_{1 l_{1}}^{t_{1 l_{1}}} \cdots \omega_{r 1}^{t_{r 1}} \omega_{r 2}^{t_{r 2}} \cdots \omega_{r l_{r}}^{t_{r l_{r}}}}{t_{11} ! t_{12} ! \cdots t_{1 l_{1}} ! \cdots t_{r 1} ! t_{r 2} ! \cdots t_{r l_{r}} !} S_{\mathfrak{R}, n_{2}, \ldots, n_{s}}^{m_{1}, \ldots, m_{s}}\left(y_{1}, \ldots, y_{s}\right) z^{\Re} \\
& \quad \times \frac{(-1)^{\Re}}{\left(1-\alpha_{1}\right)_{\mathfrak{R}-t_{11}}\left(1-\alpha_{1}\right)_{\mathfrak{R}-t_{12}} \cdots\left(1-\alpha_{1}\right)_{\mathfrak{R}-t_{1 l_{1}}} \cdots}
\end{aligned}
$$




$$
\times \frac{1}{\left(1-\alpha_{r}\right)_{\mathfrak{R}-t_{r 1}}\left(1-\alpha_{r}\right)_{\mathfrak{R}-t_{r 2}} \cdots\left(1-\alpha_{r}\right)_{\mathfrak{R}-t_{r l_{r}}}}
$$

which completes the proof.

Corollary 2.5. In view of equations (1.13) and (2.13), we have the following relation

$$
\begin{aligned}
& \sum_{n=0}^{\infty} \frac{\mathscr{U}_{n ; l_{1}, \ldots, l_{r}}^{\left(\alpha_{1}-n, \ldots, \alpha_{r}-n\right)}\left(x_{1}, \ldots, x_{r}\right)}{\prod_{i=1}^{r}\left\{\left(1-\alpha_{i}\right)_{n}\right\}^{l_{i}}} F_{C: D^{(1)}, \ldots, D^{(s)}}^{A: 1+B^{(1)}, \ldots ; 1+B^{(s)}}\left[\begin{array}{c}
{\left[(a): \theta^{(1)}, \ldots, \theta^{(s)}\right]:} \\
{\left[(c): \psi^{(1)}, \ldots, \psi^{(s)}\right]:}
\end{array}\right. \\
& \left.\begin{array}{rl}
{\left[-n ; m_{1}\right],\left[b^{(1)} ; \phi^{(1)}\right] ;} & \ldots ;\left[-n_{s} ; m_{s}\right],\left[b^{(s)} ; \phi^{(s)}\right] ; \\
-, \quad\left[d^{(1)} ; \delta^{(1)}\right] ; & \ldots ; \quad-, \quad\left[d^{(s)} ; \delta^{(s)}\right] ;
\end{array}\right] z^{n}
\end{aligned}
$$

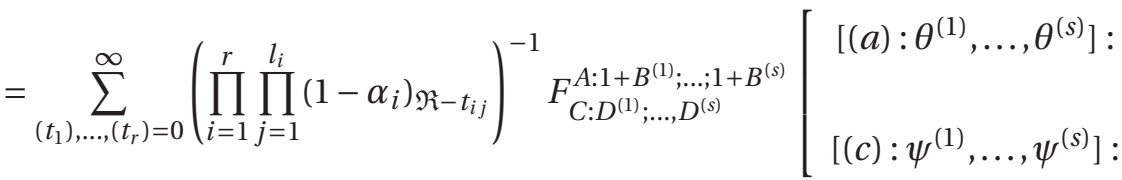

$$
\begin{aligned}
& {\left[-\mathfrak{R} ; m_{1}\right],\left[b^{(1)} ; \phi^{(1)}\right] ; \quad \ldots ;\left[-n_{s} ; m_{s}\right],\left[b^{(s)} ; \phi^{(s)}\right] ;} \\
& -, \quad\left[d^{(1)} ; \delta^{(1)}\right] ; \quad \ldots ; \quad-, \quad\left[d^{(s)} ; \delta^{(s)}\right] ; \\
& \times \frac{\left(-\omega_{11} z\right)^{t_{11}}}{t_{11} !} \cdots \frac{\left(-\omega_{1 l_{1}} z\right)^{t_{1 l_{1}}}}{t_{1 l_{1}} !} \cdots \frac{\left(-\omega_{r 1} z\right)^{t_{r 1}}}{t_{r 1} !} \cdots \frac{\left(-\omega_{r l_{r}} z\right)^{t_{r l}}}{t_{r l_{r}} !}
\end{aligned}
$$

where

$$
\left(t_{i}\right):=t_{i 1}, \ldots, t_{i l_{i}}(i=1, \ldots, r) \text { and } \Re:=\sum_{i=1}^{r} \sum_{j=1}^{l_{i}} t_{i j} .
$$

For a suitably bounded non-vanishing multiple sequence $\left\{\Lambda\left(n ; k_{1}, \ldots, k_{s}\right)\right\}_{n, k_{1}, \ldots, k_{s} \in \mathbb{N}_{0}}$ of real or complex parameters, we define a function $\Psi_{n}\left(m_{1}, \ldots, m_{s} ; y_{1}, \ldots, y_{s}\right)$ of $s$ variables where $m_{j} \in \mathbb{N}$, for $j=1, \ldots, s$, by

$$
\begin{aligned}
& \Psi_{n}\left(m_{1}, \ldots, m_{s} ; y_{1}, \ldots, y_{s}\right)=\frac{S_{n}^{m_{1}, \ldots, m_{s}}\left(y_{1}, \ldots, y_{s}\right)}{\prod_{i=1}^{r}\left\{\left(1-\alpha_{i}\right)_{n}\right\}^{l_{i}}} \\
& \quad=\sum_{k_{1}, \ldots, k_{s}=0}^{m_{1} k_{1}+\cdots+m_{s} k_{s} \leq n} \frac{(-n)_{m_{1} k_{1}+\cdots+m_{s} k_{s}}}{\prod_{i=1}^{r}\left\{\left(1-\alpha_{i}\right)_{n}\right\}^{l_{i}}} \Lambda\left(n ; k_{1}, \ldots, k_{s}\right) \frac{y_{1}^{k_{1}} \cdots y_{s}^{k_{s}}}{k_{1} ! \cdots k_{s} !} .
\end{aligned}
$$

Theorem 2.6. The following bilateral generating function holds true:

$$
\begin{gathered}
\sum_{n=0}^{\infty} \mathscr{U}_{n ; l_{1}, \ldots, l_{r}}^{\left(\alpha_{1}-n, \ldots, \alpha_{r}-n\right)}\left(x_{1}, \ldots, x_{r}\right) \Psi_{n}\left(m_{1}, \ldots, m_{s} ; y_{1}, \ldots, y_{s}\right) z^{n} \\
\quad=\sum_{\left.\left(t_{1}\right), \ldots, t_{r}\right)=0}^{\infty}\left(\prod_{i=1}^{r} \prod_{j=1}^{l_{i}}\left(1-\alpha_{i}\right)_{\mathfrak{R}-t_{i j}}\right)^{-1} S_{\Re}^{m_{1}, \ldots, m_{s}}\left(y_{1}, \ldots, y_{s}\right)
\end{gathered}
$$


where

$$
\times \frac{\left(-\omega_{11} z\right)^{t_{11}}}{t_{11} !} \cdots \frac{\left(-\omega_{1 l_{1}} z\right)^{t_{1 l_{1}}}}{t_{1 l_{1}} !} \cdots \frac{\left(-\omega_{r 1} z\right)^{t_{r 1}}}{t_{r 1} !} \cdots \frac{\left(-\omega_{r l_{r}} z\right)^{t_{r l}}}{t_{r l_{r}} !}
$$

$$
\left(t_{i}\right):=t_{i 1}, \ldots, t_{i l_{i}}(i=1, \ldots, r) \text { and } \Re:=\sum_{i=1}^{r} \sum_{j=1}^{l_{i}} t_{i j} .
$$

Proof. From (1.3), we have

$$
\begin{aligned}
& \sum_{n=0}^{\infty} \mathscr{U}_{n ; l_{1}, \ldots, l_{r}}^{\left(\alpha_{1}-n, \ldots, \alpha_{r}-n\right)}\left(x_{1}, \ldots, x_{r}\right) \Xi_{n}\left(m_{1} ; n_{2}, m_{2} ; \ldots ; n_{s}, m_{s} ; y_{1}, \ldots, y_{s}\right) z^{n} \\
& =\sum_{n=0}^{\infty} \sum_{t_{r\left(l_{r}-1\right)}=0}^{n} \cdots \sum_{t_{r 1}=0}^{t_{r 2}} \sum_{t_{(r-1) l_{r-1}}=0}^{t_{r 1}} \cdots \sum_{t_{1\left(l_{r-1}\right)}=0}^{t_{1 l_{1}}} \cdots \sum_{t_{12}=0}^{t_{13}} \sum_{t_{11}=0}^{t_{12}} \\
& \times \frac{\left(\alpha_{1}-n\right)_{t_{11}}\left(\alpha_{1}-n\right)_{t_{12}-t_{11}} \cdots\left(\alpha_{1}-n\right)_{t_{1 l_{1}}-t_{1\left(l_{1}-1\right)}} \cdots}{t_{11} !\left(t_{12}-t_{11}\right) ! \cdots\left(t_{1 l_{1}}-t_{1\left(l_{1}-1\right)}\right) ! \cdots} \\
& \times \frac{\left(\alpha_{r}-n\right)_{t_{r 1}-t_{(r-1) l_{r-1}}}\left(\alpha_{r}-n\right)_{t_{r 2}-t_{r 1}} \cdots\left(\alpha_{r}-n\right)_{t_{n}-t_{r\left(l_{r}-1\right)}}}{\left(t_{r 1}-t_{(r-1) l_{r-1}}\right) !\left(t_{r 2}-t_{r 1}\right) ! \cdots\left(n-t_{r\left(l_{r}-1\right)}\right) !} \frac{S_{n}^{m_{1}, \ldots, m_{s}}\left(y_{1}, \ldots, y_{s}\right)}{\prod_{i=1}^{r}\left\{\left(1-\alpha_{i}\right)_{n}\right\}^{l_{i}}} \\
& \times \omega_{11}^{t_{11}} \omega_{12}^{t_{12}-t_{11}} \cdots \omega_{1 l_{1}}^{t_{1 l_{1}}-t_{1\left(l_{1}-1\right)}} \cdots \omega_{r 1}^{t_{r 1}-t_{(r-1) l_{r-1}}} \omega_{r 2}^{t_{r 2}-t_{r 1}} \cdots \omega_{r l_{r}}^{n-t_{r\left(l_{r}-1\right)}} z^{n} \\
& =\sum_{n=0}^{\infty} \sum_{t_{r\left(l_{r}-1\right)}=0}^{n} \cdots \sum_{t_{r 1}=0}^{t_{r 2}} \sum_{t_{(r-1) l_{r-1}}=0}^{t_{r 1}} \cdots \sum_{t_{1\left(l_{r-1}\right)}=0}^{t_{1 l_{1}}} \cdots \sum_{t_{12}=0}^{t_{13}} \sum_{t_{11}=0}^{t_{12}} \\
& \times \frac{(-1)^{n}}{\left(1-\alpha_{1}\right)_{n-t_{11}}\left(1-\alpha_{1}\right)_{n-t_{12}+t_{11}} \cdots\left(1-\alpha_{1}\right)_{n-t_{1 l_{1}}+t_{1\left(l_{1}-1\right)}}} \cdots \\
& \times \frac{S_{n}^{m_{1}, \ldots, m_{s}}\left(y_{1}, \ldots, y_{s}\right)}{\left(1-\alpha_{r}\right)_{n-t_{r 1}+t_{(r-1) l_{r-1}}}\left(1-\alpha_{r}\right)_{n-t_{r 2}+t_{r 1}} \cdots\left(1-\alpha_{r}\right)_{t_{r\left(l_{r}-1\right)}}} \\
& \times \frac{\omega_{11}^{t_{11}} \omega_{12}^{t_{12}-t_{11}} \cdots \omega_{1 l_{1}}^{t_{1 l_{1}}-t_{1\left(l_{1}-1\right)}} \cdots \omega_{r 1}^{t_{r 1}-t_{(r-1)} l_{r-1}} \omega_{r 2}^{t_{r 2}-t_{r 1}} \cdots \omega_{r l_{r}}^{n-t_{r(l-1)}}}{t_{11} !\left(t_{12}-t_{11}\right) ! \cdots\left(t_{1 l_{1}}-t_{1\left(l_{1}-1\right)}\right) ! \cdots\left(t_{r 1}-t_{(r-1) l_{r-1}}\right) !\left(t_{r 2}-t_{r 1}\right) ! \cdots\left(n-t_{r\left(l_{r}-1\right)}\right) !} z^{n}
\end{aligned}
$$

With the help of Lemma 2.1, the result follows easily.

Corollary 2.7. In view of equations (1.12) and (2.18), we have the following relation

$$
\begin{aligned}
& \sum_{n=0}^{\infty} \frac{\mathscr{U}_{n ; l_{1}, \ldots, l_{r}}^{\left(\alpha_{1}-n, \ldots, \alpha_{r}-n\right)}\left(x_{1}, \ldots, x_{r}\right)}{\prod_{i=1}^{r}\left\{\left(1-\alpha_{i}\right)_{n}\right\}^{l_{i}}} F_{C: D^{(1)} ; \ldots, D^{(s)}}^{A+1: B^{(1)}, \ldots ; B^{(s)}}\left[\begin{array}{c}
{\left[(-n): m_{1}, \ldots, m_{s}\right],} \\
-,
\end{array}\right. \\
& \left.\begin{array}{l}
{\left[(a): \theta^{(1)}, \ldots, \theta^{(s)}\right]:\left[b^{(1)} ; \phi^{(1)}\right] ; \quad \ldots ;\left[b^{(s)} ; \phi^{(s)}\right] ;} \\
{\left[(c): \psi^{(1)}, \ldots, \psi^{(s)}\right]:\left[d^{(1)} ; \delta^{(1)}\right] ; \quad \ldots ;\left[d^{(s)} ; \delta^{(s)}\right] ;}
\end{array}\right] z^{n} \\
& =\sum_{\left(t_{1}\right), \ldots,\left(t_{r}\right)=0}^{\infty}\left(\prod_{i=1}^{r} \prod_{j=1}^{l_{i}}\left(1-\alpha_{i}\right)_{\Re-t_{i j}}\right)^{-1} F_{C: D^{(1)} ; \ldots, D^{(s)}}^{A+1:(1)}\left[\begin{array}{c}
{\left[-\Re: m_{1}, \ldots, m_{s}\right],} \\
-,
\end{array}\right.
\end{aligned}
$$




$$
\begin{aligned}
& {\left[(a): \theta^{(1)}, \ldots, \theta^{(s)}\right]:\left[b^{(1)} ; \phi^{(1)}\right] ; \quad \ldots ;\left[b^{(s)} ; \phi^{(s)}\right] ;} \\
& {\left[(c): \psi^{(1)}, \ldots, \psi^{(s)}\right]:\left[d^{(1)} ; \delta^{(1)}\right] ; \quad \ldots ;\left[d^{(s)} ; \delta^{(s)}\right] ;} \\
& \times \frac{\left(-\omega_{11} z\right)^{t_{11}}}{t_{11} !} \cdots \frac{\left(-\omega_{1 l_{1}} z\right)^{t_{1 l_{1}}}}{t_{1 l_{1}} !} \cdots \frac{\left(-\omega_{r 1} z\right)^{t_{r 1}}}{t_{r 1} !} \cdots \frac{\left(-\omega_{r l_{r}} z\right)^{t_{r l}}}{t_{r l_{r}} !}
\end{aligned}
$$

where

$$
\left(t_{i}\right):=t_{i 1}, \ldots, t_{i l_{i}}(i=1, \ldots, r) \text { and } \Re:=\sum_{i=1}^{r} \sum_{j=1}^{l_{i}} t_{i j} .
$$

Let us shift our focus on two special cases of Theorem 2.6. First of all, setting $s=1$ and using the fact that the Gould-Hopper polynomials [9, p. 58] $g_{n}^{m}(y, h)$ defined by

$$
g_{n}^{m}(y, h)=\sum_{k=0}^{\left[\frac{n}{m}\right]} \frac{n !}{k !(n-m k) !} h^{k} y^{n-m k}
$$

are related to the polynomials $S_{n}^{m}(y)$ (see [20, p.161, Eq. (1.15)]) by

$$
S_{n}^{m}(y)=(-1)^{n}\left(\frac{y}{h}\right)^{n / m} g_{n}^{m}\left(-\left(\frac{h}{y}\right)^{1 / m}, h\right) .
$$

Thus, we obtain the following relationship between the Erkuş-Srivastava polynomials and the Gould-Hopper polynomials:

$$
\begin{aligned}
& \sum_{n=0}^{\infty} \frac{\mathscr{U}_{n ; l_{1}, \ldots, l_{r}}^{\left(\alpha_{1}-n, \ldots, \alpha_{r}-n\right)}\left(x_{1}, \ldots, x_{r}\right)}{\prod_{i=1}^{r}\left\{\left(1-\alpha_{i}\right)_{n}\right\}^{l_{i}}}(-1)^{n}\left(\frac{y}{h}\right)^{n / m} g_{n}^{m}\left(-\left(\frac{h}{y}\right)^{1 / m}, h\right) z^{n} \\
& =\sum_{\left.\left(t_{1}\right), \ldots, t_{r}\right)=0}^{\infty}\left(\prod_{i=1}^{r} \prod_{j=1}^{l_{i}}\left(1-\alpha_{i}\right)_{\mathfrak{R}-t_{i j}}\right)^{-1}\left(\frac{y}{h}\right)^{\frac{\mathfrak{R}}{m}} g_{\mathfrak{R}}^{m}\left(-\left(\frac{h}{y}\right)^{1 / m}, h\right) \\
& \quad \times \frac{\left(-\omega_{11} z\right)^{t_{11}}}{t_{11} !} \cdots \frac{\left(-\omega_{1 l_{1}} z\right)^{t_{1 l_{1}}}}{t_{1 l_{1}} !} \cdots \frac{\left(-\omega_{r 1} z\right)^{t_{r 1}}}{t_{r 1} !} \cdots \frac{\left(-\omega_{r l_{r}} z\right)^{t_{r l}}}{t_{r l_{r}} !}
\end{aligned}
$$

where, as seen previously,

$$
\left(t_{i}\right):=t_{i 1}, \ldots, t_{i l_{i}}(i=1, \ldots, r) \text { and } \Re:=\sum_{i=1}^{r} \sum_{j=1}^{l_{i}} t_{i j} .
$$

Next, putting $s=1, y_{1}=u$ and considering the relation established by Srivastava and Singh [20, p. 160, Eq. (1.13)] between the generalized Bessel polynomials $y_{n}(y, \gamma, \beta)$ introduced by Krall and Frink [11, p. 108, Eq. (34)] and defined by

$$
y_{n}(y, \gamma, \beta)=\sum_{k=0}^{n}\left(\begin{array}{l}
n \\
k
\end{array}\right)\left(\begin{array}{c}
n+\gamma+k-2 \\
k
\end{array}\right) k !\left(\frac{y}{\beta}\right)^{k}
$$


and the Srivastava polynomials $S_{n}^{m}(y)$, namely,

$$
S_{n}^{1}(y)=y_{n}(-\beta y, \gamma, \beta) .
$$

This relation is obtained by replacing $A_{n, k}$ by $(\gamma+n-1)_{k}$ and $m$ by 1 in (1.7). Therefore, we have the next relation between the Chan-Chyan-Srivastava polynomials and the generalized Bessel polynomials:

$$
\begin{aligned}
& \sum_{n=0}^{\infty} \frac{\mathscr{U}_{n ; l_{1}, \ldots, l_{r}}^{\left(\alpha_{1}-n, \ldots, \alpha_{r}-n\right)}\left(x_{1}, \ldots, x_{r}\right)}{\prod_{i=1}^{r}\left\{\left(1-\alpha_{i}\right)_{n}\right\}^{l_{i}}} y_{n}(-\beta u, \gamma, \beta) z^{n} \\
& =\sum_{\left(t_{1}\right), \ldots,\left(t_{r}\right)=0}^{\infty}\left(\prod_{i=1}^{r} \prod_{j=1}^{l_{i}}\left(1-\alpha_{i}\right)_{\mathfrak{R}-t_{i j}}\right)^{-1} y_{\mathfrak{R}}(-\beta u, \gamma, \beta) \\
& \quad \times \frac{\left(-\omega_{11} z\right)^{t_{11}}}{t_{11} !} \cdots \frac{\left(-\omega_{1 l_{1}} z\right)^{t_{1 l_{1}}}}{t_{1 l_{1}} !} \cdots \frac{\left(-\omega_{r 1} z\right)^{t_{r 1}}}{t_{r 1} !} \cdots \frac{\left(-\omega_{r l_{r}} z\right)^{t_{r l}}}{t_{r l_{r}} !} .
\end{aligned}
$$

We end this paper by giving a bilateral generating function involving the class of polynomials $P_{n}^{m, N_{1}, \ldots, N_{r-1}}\left(x_{1}, \ldots, x_{r}\right)$ defined by (1.14). For a suitably bounded non-vanishing multiple sequence $\left\{A_{m+n, k_{1}, \ldots, k_{r-1}}\right\}_{n, m, k_{1}, \ldots, k_{r-1} \in \mathbb{N}_{0}}$ of real or complex parameters, we define a function $\Delta_{n}\left(m, N_{1}, \ldots, N_{s-1} ; y_{1}, \ldots, y_{s}\right)$ of $s$-variables where $N_{j} \in \mathbb{N}$, for $j=1, \ldots, s-1$, by

$$
\begin{aligned}
& \Delta_{n}\left(m, N_{1}, \ldots, N_{s-1} ; y_{1}, \ldots, y_{s}\right)=\frac{P_{n}^{m, N_{1}, \ldots, N_{s-1}}\left(y_{1}, \ldots, y_{s}\right)}{\prod_{i=1}^{r}\left\{\left(1-\alpha_{i}\right)_{n}\right\}^{l_{i}}} \\
& \quad=\sum_{k_{s-1}=0}^{\left[\frac{n}{N_{s-1}}\right]\left[\frac{k_{s-1}}{N_{s-2}}\right]} \cdots \sum_{k_{s-2}=0}^{\left[\frac{k_{3}}{N_{2}}\right]\left[\frac{k_{2}}{N_{1}}\right]} \frac{A_{m+n, k_{1}, \ldots, k_{s-1}}}{\sum_{k_{2}=0}^{k_{1}}} \frac{y_{1}^{k_{1}} y_{2}^{k_{2}-N_{1} k_{1}} \cdots y_{s}^{n-N_{s-1} k_{s-1}}}{\prod_{i=1}^{r}\left\{\left(1-\alpha_{i}\right)_{n}\right\}_{i}^{l_{i}}} \frac{k_{1} !\left(k_{2}-N_{1} k_{1}\right) ! \cdots\left(n-N_{s-1} k_{s-1}\right) !}{}
\end{aligned}
$$

Theorem 2.8. The following bilateral generating function holds true:

$$
\begin{aligned}
\sum_{n=0}^{\infty} \mathscr{U}_{n ; l_{1}, \ldots, l_{r}}^{\left(\alpha_{1}-n, \ldots, \alpha_{r}-n\right)}\left(x_{1}, \ldots, x_{r}\right) \Delta_{n}\left(m, N_{1}, \ldots, N_{s-1} ; y_{1}, \ldots, y_{s}\right) z^{n} \\
=\sum_{\left(t_{1}\right), \ldots,\left(t_{r}\right)=0}^{\infty}\left(\prod_{i=1}^{r} \prod_{j=1}^{l_{i}}\left(1-\alpha_{i}\right)_{\Re-t_{i j}}\right)^{-1} P_{\Re}^{m, N_{1}, \ldots, N_{s-1}}\left(y_{1}, \ldots, y_{s}\right) \\
\quad \times \frac{\left(-\omega_{11} z\right)^{t_{11}}}{t_{11} !} \cdots \frac{\left(-\omega_{1 l_{1}} z\right)^{t_{1 l_{1}}}}{t_{1 l_{1}} !} \cdots \frac{\left(-\omega_{r 1} z\right)^{t_{r 1}}}{t_{r 1} !} \cdots \frac{\left(-\omega_{r l_{r}} z\right)^{t_{r l}}}{t_{r l_{r}} !}
\end{aligned}
$$

where

$$
\left(t_{i}\right):=t_{i 1}, \ldots, t_{i l_{i}}(i=1, \ldots, r) \text { and } \Re:=\sum_{i=1}^{r} \sum_{j=1}^{l_{i}} t_{i j} .
$$

Proof. The proof is omitted since it is almost the same as the one of Theorem 2.6.

Setting $N_{1}=N_{2}=\cdots=N_{s-1}=1$ and

$$
A_{m+n, k_{1}, \ldots, k_{s-1}}=\left(\alpha_{1}\right)_{k_{1}}\left(\alpha_{2}\right)_{k_{2}-k_{1}} \cdots\left(\alpha_{s-1}\right)_{k_{s-1}-k_{s-2}}\left(\alpha_{s}\right)_{m}\left(\alpha_{s}+m\right)_{n-k_{s-1}},
$$


we find from [10, p. 627, Eq. (1.7)] that

$$
P_{n}^{m, 1, \ldots, 1}\left(y_{1}, \ldots, y_{s}\right)=\left(\alpha_{s}\right)_{m} g_{n}^{\left(\alpha_{1}, \ldots, \alpha_{s}+m\right)}\left(y_{1}, \ldots, y_{s}\right)
$$

Putting $s=r, m=0$ and substituting $x_{j}=y_{j}$, for $j=1, \ldots, r$, we obtain the next relation.

Corollary 2.9. The following bilateral generating function involving the product of the ErkuşSrivastava polynomials and the Chan-Chyan-Srivastava polynomials $g_{n}^{\left(\alpha_{1}, \ldots, \alpha_{r}\right)}\left(x_{1}, \ldots, x_{r}\right)$ holds true:

$$
\begin{aligned}
\sum_{n=0}^{\infty} & \frac{\mathcal{U}_{n ; l_{1}, \ldots, l_{r}}^{\left(\alpha_{1}-n, \ldots, \alpha_{r}-n\right)}\left(x_{1}, \ldots, x_{r}\right) g_{n}^{\left(\alpha_{1}, \ldots, \alpha_{r}\right)}\left(x_{1}, \ldots, x_{r}\right)}{\prod_{i=1}^{r}\left\{\left(1-\alpha_{i}\right)_{n}\right\}^{l_{i}}} z^{n} \\
= & \sum_{\left(t_{1}\right), \ldots,\left(t_{r}\right)=0}^{\infty}\left(\prod_{i=1}^{r} \prod_{j=1}^{l_{i}}\left(1-\alpha_{i}\right)_{\mathfrak{R}-t_{i j}}\right)^{-1} g_{\mathfrak{R}}^{\left(\alpha_{1}, \ldots, \alpha_{r}\right)}\left(x_{1}, \ldots, x_{r}\right) \\
& \times \frac{\left(-\omega_{11} z\right)^{t_{11}}}{t_{11} !} \cdots \frac{\left(-\omega_{1 l_{1}} z\right)^{t_{1 l_{1}}}}{t_{1 l_{1}} !} \cdots \frac{\left(-\omega_{r 1} z\right)^{t_{r 1}}}{t_{r 1} !} \cdots \frac{\left(-\omega_{r l_{r}} z\right)^{t_{r l}}}{t_{r l_{r}} !} .
\end{aligned}
$$

Remark 2.10. In every Theorems and Corollaries obtained in this paper, we can specialize the Erkuş-Srivastava polynomials to obtain the corresponding bilateral generating functions for the Lagrange-Hermite polynomials and the Chan-Chyan-Srivastava polynomials. Note that recently, the authors in [8] gave the ones involving the Chan-Chyan-Srivastava polynomials.

\section{References}

[1] A. Altin and E. Erkuş, On a multivariable extension of the Lagrange-Hermite polynomials, Integral Transform Spec. Funct., 17(2006), 239-244.

[2] W.-C. C. Chan and C.-J. Chyan and H. M. Srivastava, The Lagrange polynomials in several variables, Integral Transform Spec. Funct., 12(2001), 139-148.

[3] K.-Y. Chen and S.-J. Liu and H.M. Srivastava, Some new results for the Lagrange polynomials in several variables, ANZIAM J., 49(2007), 243-258.

[4] G. Dattoli and P. E. Ricci and C. Cesarano, The Lagrange polynomials, the associated generalizations, and the umbral calculus, Integral Transform Spec. Funct., 14(2003), 181-186.

[5] A. Erdelyi, W. Magnus, F. Oberhettinger and F. Tricomi, Higher Transcendental Functions, vols.1-3, McGrawHill, New York, 1953.

[6] E. Erkuş, O. Duman and H. M. Srivastava, Statistical approximation of certain positive linear operators constructed by means of the Chan-Chyan-Srivastava polynomials, Appl. Math. Comput, 182(2006), $213-222$.

[7] E. Erkuş and H.M. Srivastava, A unified presentation of some families of multivariable polynomials, Integral Transform Spec. Funct., 17(2006), 267-273.

[8] S. Gaboury, R. Tremblay, M. A. Özarslan, Some bilateral generating functions involving the Chan-ChyanSrivastava polynomials and some general classes of multivariable polynomials, Comm. Korean Math. Soc., to appear, 13 pp.

[9] H. W. Gould and A. T. Hopper, Operational formulas connected with two generalizations of Hermite polynomials, Duke Math. J., 29 (1962), 51-63. 
[10] C. Kaanoğlu and M. A. Özarslan, Two-sided generating functions for certain class of $r$-variable polynomials, Mathematical and Computer Modelling, 54(2011), 625-631.

[11] H. L. Krall and O. Frink, A new class of orthogonal polynomials: the Bessel polynomials, Trans. Amer. Math. Soc., 65 (1949), 100-115.

[12] S.-J. Liu, Bilateral generating function for the Lagrange polynomials and the Lauricella functions, Integral Transform Spec. Funct., 20 (2009), 519-527.

[13] S.-J. Liu, C.-J. Chyan, H.-C. Lu and H. M. Srivastava, Bilateral generating functions for the Chan-ChyanSrivastava polynomials and the generalized Lauricella functions, Integral Transform Spec. Funct., 23 (2012), 539-549.

[14] S.-J. Liu, S.-D. Lin, H. M. Srivastava and M.-M. Wong, Bilateral generating functions for the Erkuş-Srivastava polynomials and the generalized Lauricella functions, Appl. Math. Comput, 218(2012), 7685-7693.

[15] H. M. Srivastava, A contour integral involving Fox's H-function, Indian J. Math., 14 (1972), 1-6.

[16] H. M. Srivastava, A multilinear generating function for the Konhauser sets of biorthogonal polynomials suggested by the Laguerre polynomials, Pacific J. Math., 117 (1985), 183-191.

[17] H. M. Srivastava and M. Garg, Some integrals involving a general class of polynomials and multivariable $H$ function, Rev. Roumanie Phys., 32 (1987), 685-692.

[18] H. M. Srivastava and P. W. Karlsson, Multiple Gaussian Hypergeometric Series, Halsted Press (Ellis Horwoor Limited), John Wiley and Sons, Chichester, New York, 1985.

[19] H. M. Srivastava and M. A. Özarslan and C. Kaanoğlu, Some families of generating functions for a certain class of three-variable polynomials, Integral Transform Spec. Funct., 21 (2010), 885-896.

[20] H. M. Srivastava and N. P. Singh, The integration of certain products of the multivariable H-function with a general class of polynomials, Rend. Circ. Mat. Palermo, 32 (1983), 157-187.

[21] G. Szëgo, Orthogonal polynomials, Amer. Math. Soc. Colloq. Publ., Vol. 23 Fourth edition, 1975.

Department of Mathematics and Computer Science, University of Quebec at Chicoutimi, Quebec, Canada, G7H 2B1.

E-mail: slgabour@uqac.ca

Department of Mathematics and Computer Science, University of Quebec at Chicoutimi, Quebec, Canada, G7H $2 \mathrm{~B} 1$.

E-mail: rtrembla@uqac.ca

Eastern Mediterranean University, Gazimagusa, TRNC, Mersin 10, Turkey.

E-mail: mehmetali.ozarslan@emu.edu.tr 